\title{
Faint stellar systems in the Virgo cluster: ultracompact dwarfs and globular clusters
}

\author{
Monica Haşegan ${ }^{1,2}$ and Patrick Côté en $^{1,3}$ \\ ${ }^{1}$ Department of Physics and Astronomy, Rutgers, The State University of New Jersey, \\ Piscataway, New Jersey 08854, USA \\ email: mhasegan@physics.rutgers.edu \\ ${ }^{2}$ Institute for Space Sciences, P.O.Box MG-23, RO 77125, Bucharest-Magurele, Romania \\ ${ }^{3}$ Herzberg Institute of Astrophysics, National Research Council of Canada, 5071 West Saanich \\ Road, Victoria, BC, V9E 287, Canada \\ email: Patrick.Cote@nrc-cnrc.gc.ca
}

\begin{abstract}
We examine the properties of ten compact objects in the vicinity of M87 using $H S T$ imaging and Keck spectroscopy. We find three objects to be larger and brighter than typical globular clusters; they resemble the nuclei of nucleated dEs in Virgo, having similar massto-light ratios, luminosities and colors. Their properties are consistent with models of tidally stripping of nucleated dEs. One object could be an old "stellar supercluster", formed through the amalgamation of multiple young massive clusters. Two other objects appear to be massive versions of otherwise "normal" globular clusters.
\end{abstract}

Keywords. galaxies: clusters: individual (Virgo) - galaxies: star clusters - galaxies: dwarf galaxies: formation - galaxies: kinematics and dynamics

\section{Introduction and Sample Selection}

We are carrying out a program to study the structural, photometric and dynamical properties of faint stellar systems in the Virgo Cluster. Here we present results from a recent paper (Haşegan et al. 2005) where we have examined the properties of ultracompact dwarf (UCD) galaxies in the Virgo Cluster. UCDs are a potentially new type of faint and compact stellar system which were first identified in the Fornax Cluster (see Drinkwater et al. 2003 and references therein). They may represent a missing link between dwarf elliptical galaxies and globular clusters.

Based on a combination of ground- and space-based imaging surveys, we identified several compact objects with $-11.8 \leqslant \mathrm{M}_{V} \leqslant-10.8$ in the vicinity of M87, which we provisionally classify as "Dwarf Globular Transition Objects" (DGTOs). Our analysis is based on imaging from HST - taken as part of the ACS Virgo Cluster Survey (ACSVCS; Côté et al. 2004) or from the HST/WFPC2 archive - and high-resolution spectroscopy from Keck/ESI.

\section{Results and conclusions}

The left panel of Figure 1 shows a finding chart for three DGTOs (S928, S999 and H8005) near M87, along with two additional candidates without ESI spectra. The $0.75^{\prime \prime} \times$ $20^{\prime \prime}$ ESI slit used for the Keck observations is shown by the rectangles.

The right panel of Figure 1 compares the properties of these DGTOs with those of other low-mass stellar systems. From top to bottom, central velocity dispersion, half-light radius and mass surface density averaged within the half-light radius are plotted against 
total mass. Among the sample of six DGTOs shown in this figure (squares), we find two objects which are likely massive M87 globular clusters (i.e., those lying on the solid lines which represent the scaling relations of Galactic globular clusters), three objects which are probable UCDs (i.e., those clustered together with the Fornax UCDs and with the simulated tidally stripped cores of dE,Ns; Bekki et al. 2003 ), and one possible UCD (i.e., the object near the simulated merged massive young clusters; Fellhauer \& Kroupa 2002).
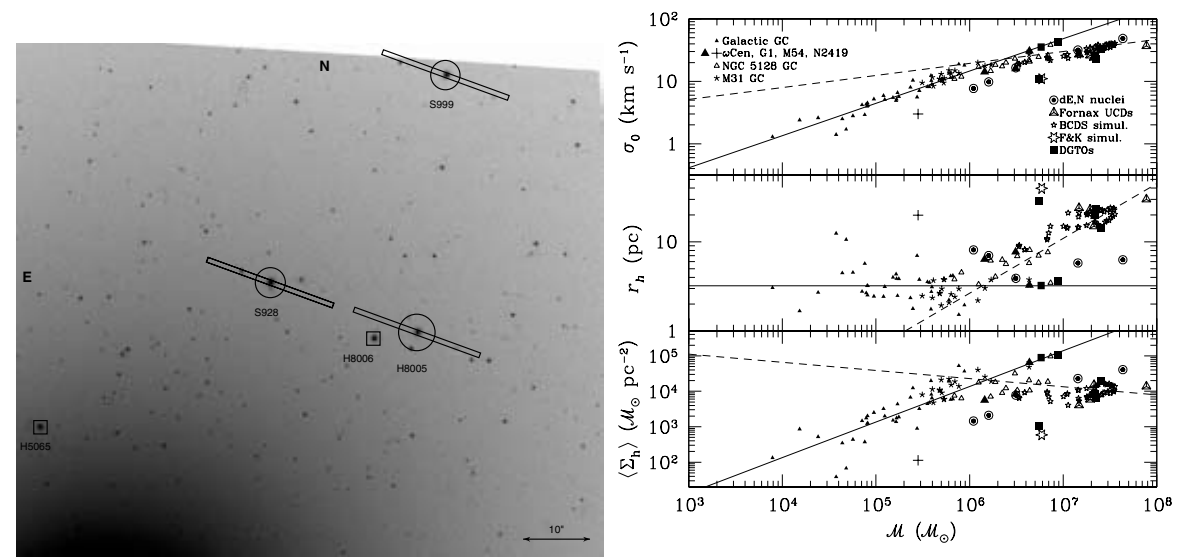

Figure 1. Left: ACSVCS image of M87 showing the location of several Dwarf Globular Transition Objects (DGTOs). Right: Scaling relations for low-mass, hot stellar systems. (Figures taken from Haşegan et al. 2005)

The UCDs are larger than typical globular clusters (with half-light radii of $\mathrm{r}_{h} \approx 20 \mathrm{pc}$ rather than $\approx 3 \mathrm{pc}$,) yet much more compact than normal dEs. Moreover, with massto-light ratios in the range $6 \leqslant M / L_{V} \leqslant 9$, they appear to contain dark matter, which would suggest a fundamental difference from globular clusters. They also bear a strong resemblance in terms of size, luminosity and mass-to-light ratio to the nuclei of dwarf elliptical galaxies in Virgo (Geha et al. 2002), supporting the identification of at least some UCDs with the tidally-stripped remains of nucleated dEs (Bekki et al. 2003) although other scenarios can not be completely ruled out. Future analysis of an extended sample of UCDs from the ACSVCS, together with nucleated dwarfs and their nuclei, should allow a greater insights into the origin of these faint stellar systems.

\section{Acknowledgements}

These results are part of a collaborative effort by the ACSVCS team, whom we thank.

\section{References}

Bekki, K., Couch, W.J., Drinkwater, M.J. \& Shioya, Y. 2003, MNRAS 346, 11

Côté, P., Blakeslee, J.P., Ferrarese, L., Jordán, A., Mei, S., Merritt, D., Milosavljević, M., Peng, E., Tonry, J.L. \& West, M.J. 2004, ApJS 153, 223

Drinkwater, M.J., Gregg, M.D., Hilker, M., Bekki, K., Couch, W.J., Ferguson, H.C., Jones, J.B. \& Phillipps, S. 2003, Nature 423, 519

Fellhauer, M. \& Kroupa, P. 2002, ApJ 124, 2006

Geha, M., Guhathakurta, P. \& van der Marel, R.P. 2002, AJ 124, 3073

Haşegan, M., Jordan, A., Côté, P., Djorgovski, S.G., McLaughlin, D.E., Blakeslee, J.P., Mei, S., West, M.J., Peng, E.W., Ferrarese, L., Milosavljević, M., Tonry, J.L. \& Merritt, D. 2005, ApJ 627, 203 